University of Nebraska - Lincoln

DigitalCommons@University of Nebraska - Lincoln

US Army Research

U.S. Department of Defense

2011

Dislocation-twin interactions in nanocrystalline fcc metals

Y. T. Zhu

North Carolina State University at Raleigh, ytzhu@ncsu.edu

X. L. Wu

Chinese Academy of Sciences

X. Z. Liao

University of Sydney

J. Narayan

North Carolina State University at Raleigh

L. J. Keckés

US Army Research Laboratory

See next page for additional authors

Follow this and additional works at: https://digitalcommons.unl.edu/usarmyresearch

Part of the Operations Research, Systems Engineering and Industrial Engineering Commons

Zhu, Y. T.; Wu, X. L.; Liao, X. Z.; Narayan, J.; Keckés, L. J.; and Mathaudhu, S. N., "Dislocation-twin interactions in nanocrystalline fcc metals" (2011). US Army Research. 160.

https://digitalcommons.unl.edu/usarmyresearch/160

This Article is brought to you for free and open access by the U.S. Department of Defense at DigitalCommons@University of Nebraska - Lincoln. It has been accepted for inclusion in US Army Research by an authorized administrator of DigitalCommons@University of Nebraska - Lincoln. 
Authors

Y. T. Zhu, X. L. Wu, X. Z. Liao, J. Narayan, L. J. Keckés, and S. N. Mathaudhu 


\title{
Dislocation-twin interactions in nanocrystalline fcc metals
}

\author{
Y.T. Zhu ${ }^{\text {a,* }}$, X.L. Wu ${ }^{\text {b }}$, X.Z. Liao ${ }^{\text {c }}$, J. Narayan ${ }^{\text {a }}$, L.J. Kecskés ${ }^{\text {d }}$, S.N. Mathaudhu ${ }^{d}$ \\ ${ }^{a}$ Department of Materials Science and Engineering, North Carolina State University, Raleigh, NC 27695, USA \\ ${ }^{\mathrm{b}}$ State Key Laboratory of Nonlinear Mechanics, Institute of Mechanics, Chinese Academy of Sciences, Beijing 100190, People's Republic of China \\ ${ }^{\mathrm{c}}$ School of Aerospace, Mechanical and Mechatronic Engineering, University of Sydney, Sydney, NSW 2006, Australia \\ ${ }^{\mathrm{d}}$ US Army Research Laboratory, Aberdeen Proving Ground, MD 21005, USA
}

Received 16 August 2010; received in revised form 7 October 2010; accepted 9 October 2010

\begin{abstract}
Dislocation interaction with and accumulation at twin boundaries have been reported to significantly improve the strength and ductility of nanostructured face-centered cubic (fcc) metals and alloys. Here we systematically describe plausible dislocation interactions at twin boundaries. Depending on the characteristics of the dislocations and the driving stress, possible dislocation reactions at twin boundaries include cross-slip into the twinning plane to cause twin growth or de-twinning, formation of a sessile stair-rod dislocation at the twin boundary, and transmission across the twin boundary. The energy barriers for these dislocation reactions are described and compared.
\end{abstract}

(C) 2010 Acta Materialia Inc. Published by Elsevier Ltd. All rights reserved.

Keywords: Nanocrystalline materials; Dislocations; Twin boundaries; Interactions; fcc

\section{Introduction}

Twins have been reported to significantly affect the mechanical properties of nanostructured face-centered cubic (fcc) metals and alloys [1-10]. Importantly, twins have been shown to be able to simultaneously increase the strength and ductility of nanostructured metals, which is attributed to the dislocation interaction with and accumulation at twin boundaries. Since deformation twinning usually occurs simultaneously with the slip of perfect and partial dislocations, interactions between twins and gliding dislocations inevitably occur at twin boundaries. Nanocrystalline fcc metals have been found to deform via twinning more readily than their coarse grained counterparts [11-22]. This increases the probability of interactions between dislocations and twins in nanocrystalline fcc metals. Furthermore, nanocrystalline fcc metals usually have high strength, and the resulting high flow stress may provide enough driving force to activate some energetically

\footnotetext{
* Corresponding author. Tel.: +1 919513 0559; fax: +1 9195153419.

E-mail address: ytzhu@ncsu.edu (Y.T. Zhu).
}

unfavorable dislocation reactions at the twin boundaries. Therefore, it is of scientific interest and practical importance to understand how the dislocations react at twin boundaries.

Dislocation reactions at twin boundaries have been observed both experimentally $[2,3,23-32]$ and by molecular dynamics (MD) simulations [33-41]. However, no systematic investigation has been reported. It is the objective of this paper to systematically describe plausible dislocation reactions at twin boundaries.

\section{Possible dislocations to react with twin boundaries}

There are only four types of dislocations in the fcc structure that may react at a twin boundary. They can be described with the assistance of Fig. 1, which shows a Thompson tetrahedron on the twin boundary. Note that the twin boundary is also the (llll 111$)$ slip plane, i.e. the ABC plane in the Thompson tetrahedron. The other three slip planes on the tetrahedron are the $A C D, A B D$ and BCD planes. Before a dislocation reacts at a twin boundary it first needs to slip on one of these three planes. When the 


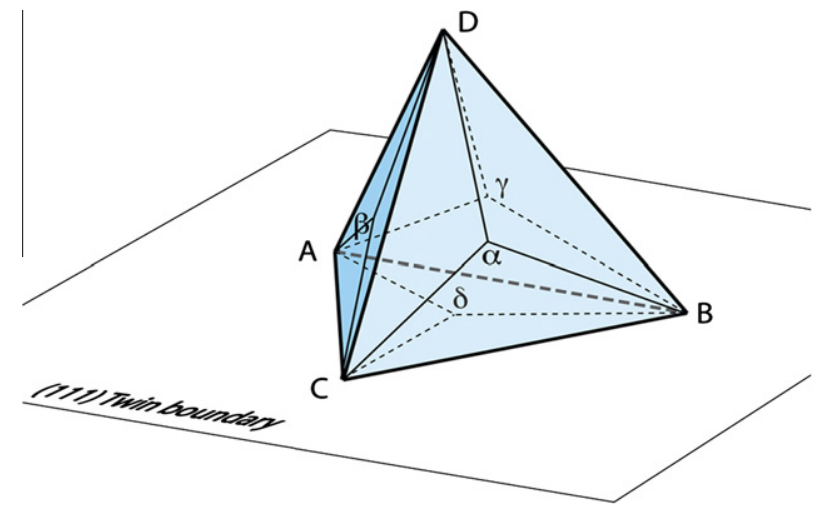

Fig. 1. Illustration of a Thompson tetrahedron on the coherent twin boundary, a ( $\left.\begin{array}{lll}1 & 1 & 1\end{array}\right)$ slip plane, which coincides with the ABC plane in the Thompson tetrahedron.

dislocation reaches the twin boundary the dislocation line should become parallel to the intersection line of the slip plane and the twin boundary, which are either $\mathrm{AB}, \mathrm{BC}$ or CA. Since the three slip planes are identical close-packed planes to gliding dislocations due to the crystal symmetry for dislocation slip, we need to consider only one representative plane hereafter.

To make the discussion easier, we unfold the Thompson tetrahedron onto a two-dimensional representation, as shown in Fig. 2. Assuming that a dislocation glides on the $\mathrm{BCD}$, i.e. $(\overline{1} 1 \overline{1})$, plane toward the twin boundary, the orientation of the dislocation line will become parallel to $\mathrm{BC}$ when it reaches the twin boundary. From Figs. 1 and 2 it can be deduced that this dislocation can be one of four possible types: (a) a $30^{\circ}$ Shockley partial dislocation, i.e. the partial's Burgers vector is at a $30^{\circ}$ angle to the dislocation line; (b) a $90^{\circ}$ Shockley partial dislocation; (c) a screw perfect dislocation with the Burgers vector parallel to the

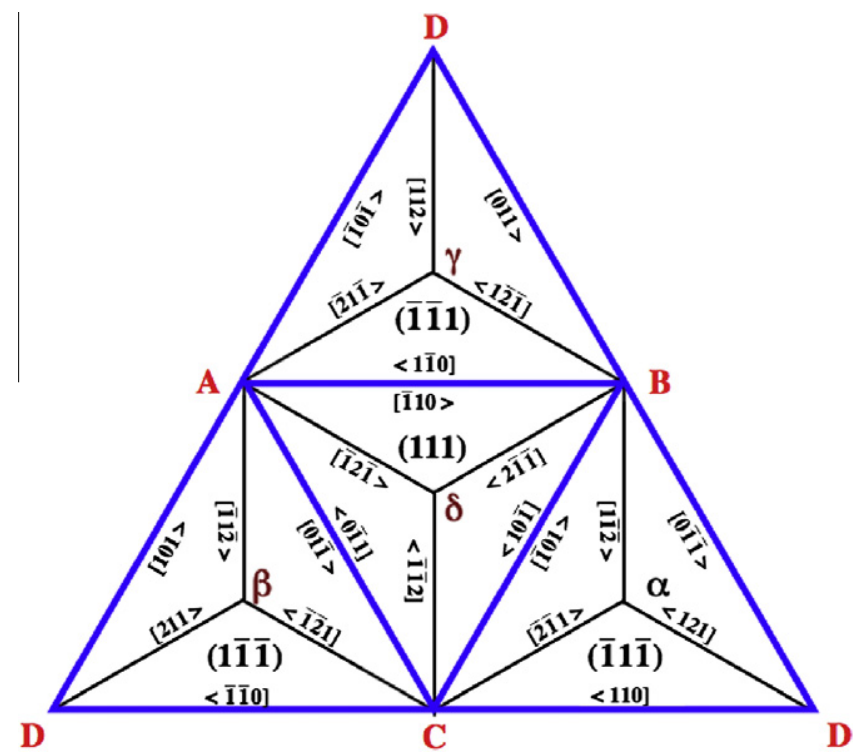

Fig. 2. Two-dimensional representation of the Thompson tetrahedron illustrating the possible slip planes and the Burgers vectors of dislocations in an fcc crystal. dislocation line; (d) a $60^{\circ}$ perfect dislocation. The dislocation reaction at the twin boundary is determined by both the type of dislocation and the magnitude and orientation of the applied stress. In the following sections we will describe possible reactions of each type of dislocation at the twin boundary.

\section{A $30^{\circ}$ partial at the twin boundary}

Assuming that a $30^{\circ}$ Shockley partial dislocation $\mathbf{B} \boldsymbol{\alpha}$ glides on the BCD plane towards a twin boundary, at the twin boundary it may either cross-slip into the twin boundary plane (ABC plane) or be transmited across the twin boundary to release a dislocation on the other side of the twin boundary. Below we describe the dislocation reactions for these two scenarios.

\subsection{Cross-slip of the $30^{\circ}$ partial at the twin boundary}

When the $30^{\circ}$ Shockley partial dislocation cross-slips into the $\mathrm{ABC}$ plane at the twin boundary, it can react at the twin boundary to grow a twin [42]. Similarly, it should also be able to cause de-twinning by moving the twin boundary towards the twin interior. The twin growth process has been described in a previous paper [42]. The de-twinning process is very similar to the twin growth process, except that the partial glides in the opposite direction after cross-slip. Here we also adopt the convention used in Fig. 2 of Zhu et al. [42], and will describe the de-twinning process only.

Fig. 3 illustrates the de-twinning process caused by the interaction of a $30^{\circ}$ partial, $\mathrm{B} \alpha$, with the twin boundary. Fig. 3a illustrates the partial $\mathbf{B} \boldsymbol{\alpha}$ slip on the BCD plane, leaving behind a stacking fault (SF), and stopped at the twin boundary, TB, represented by a thick black line. Under appropriate applied stress the following dislocation reaction occurs (see Figs. 1 and 2):

$\mathbf{B} \boldsymbol{\alpha} \rightarrow \mathbf{B} \boldsymbol{\delta}+\boldsymbol{\delta} \boldsymbol{\alpha}$

If $\mathbf{B} \boldsymbol{\delta}$ glides to the left it will move the twin boundary towards the twin interior by one atomic plane. This leaves a step and a stair-rod dislocation at the twin boundary.

The stair-rod dislocation $\boldsymbol{\delta} \boldsymbol{\alpha}$ could further dissociate into two partial dislocations according to the Thompson tetrahedron:

$\delta \boldsymbol{\alpha} \rightarrow \boldsymbol{\delta} \mathbf{B}+\mathbf{B} \boldsymbol{\alpha}$

where the partial $\delta \mathrm{B}$ will glide on the twin boundary in the opposite direction to $\mathbf{B} \boldsymbol{\delta}$ under the same applied stress, because $\boldsymbol{\delta} \mathbf{B}$ and $\mathbf{B} \boldsymbol{\delta}$ represent partials with opposite Burgers vectors.

As shown in Fig. 3c, after the $\boldsymbol{\delta} \mathbf{B}$ glides to the right the twin thickness is reduced by one atomic plane, i.e. de-twinning occurred during the process. The partial $\mathbf{B} \boldsymbol{\alpha}$ advances to the new twin boundary under the original applied stress, and can repeat the above process to annihilate the whole twin. 

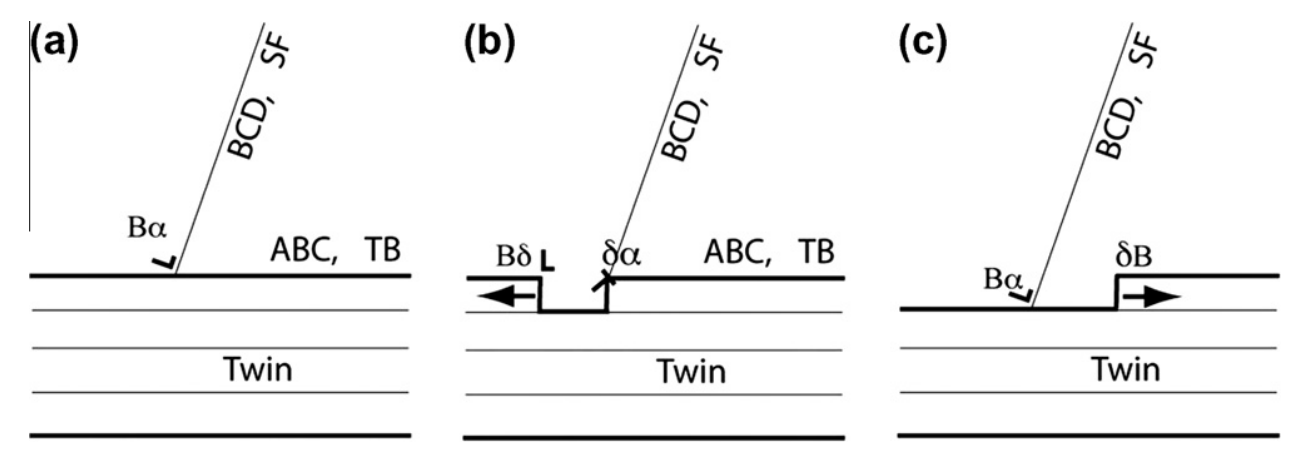

Fig. 3. De-twinning process caused by the interaction of a $30^{\circ}$ partial, $\mathrm{B} \alpha$, with the twin boundary. SF marks the stacking fault, TB marks the twin boundary (thick black lines). $\mathrm{BCD}$ and $\mathrm{ABC}$ represent the $\mathrm{BCD}$ and $\mathrm{ABC}$ slip planes in the Thompson tetrahedron, respectively.

There is an energy barrier to the dislocation reactions described in Eqs. (1) and (2), because the reactions increase the total energy of the dislocations. To estimate the energy barrier we invoke the isotropic elastic dislocation energy per unit dislocation line length [43]:

$E_{\beta}=\frac{G b^{2}\left(1-v \cos ^{2} \beta\right)}{4 \pi(1-v)} \ln \frac{R}{r_{0}}$

where $E_{\beta}$ is the energy per unit length of a dislocation with a Burgers vector is at an angle $\beta$ to the dislocation line, $G$ is the shear modulus, $v$ is the Poisson's ratio, $R$ can be estimated as the grain size $d$, and $r_{0}$ can be estimated as the Burgers vector $b$ [44]. Eq. (3) can be rewritten as:

$E_{\beta}=\frac{G b^{2}\left(1-v \cos ^{2} \beta\right)}{4 \pi(1-v)} \ln \frac{d}{b}$

The dislocation core energy is not included in Eq. (4). For covalent and ionic crystals, the core energy is relatively high and also a strong function of orientation $[45,46]$. For metals with a close-packed structure, such as fcc metals, the core energy is about $0.1-0.05 G b^{2}$. The calculation of core energies of a dislocation is still a topic of computational and analytical study. One way to incorporate the core energy into Eq. (4) is to choose a smaller $r_{0}$, i.e. $r_{0}=b / \alpha$, where $\alpha$ can be calculated as [45]:

$\alpha=\frac{\sqrt{3} e^{1+\gamma}(1-v)}{\sqrt{2}\left[\sin ^{2} \beta+e^{\gamma}(1-v) \cos ^{2} \beta\right]}$

where $\gamma=(1-2 v) / 4(1-v)$. Therefore, the total dislocation energy can be described as:

$E_{\beta}=\frac{G b^{2}\left(1-v \cos ^{2} \beta\right)}{4 \pi(1-v)} \ln \frac{\alpha d}{b}$

Note that for fcc metals the magnitudes of the Burgers vector are $a / \sqrt{ } 2$ for a perfect dislocation, $a / \sqrt{ } 6$ for a partial dislocation, and $a / 3 \sqrt{ } 2$ for the stair-rod dislocation $\delta \boldsymbol{\alpha}$. These quantities will be used to estimate the dislocation energy changes for dislocation reactions.

The energy increase (energy barrier) for a dislocation reaction can be calculated by subtracting the total energy of the initial dislocations from that of the dislocation(s) produced by the reaction. For example, for the reaction described in Eq. (1) the energy barrier can be calculated as:
$\Delta E_{e q .1}=E_{\mathbf{B} \delta}+E_{\delta \alpha}-E_{\mathbf{B} \alpha}$

According to the Thompson tetrahedron both $\mathbf{B} \boldsymbol{\alpha}$ and $\mathrm{B} \delta$ are $30^{\circ}$ partials, i.e. $\beta=30^{\circ}$, while the stair-rod dislocation $\delta \boldsymbol{\alpha}$ is an edge dislocation $\left(\beta=90^{\circ}\right)$. Substituting Eq. (6) into Eq. (7) and using the appropriate magnitudes of Burgers vectors, the energy barrier for the dislocation reaction in Eq. (1) can be calculated as:

$\Delta E_{E q 1}^{B \alpha \rightarrow B \delta+\delta \alpha}=\frac{G a^{2}}{72 \pi(1-v)} \ln \frac{\sqrt{2} d}{a}+\frac{G a^{2}}{72 \pi(1-v)} \ln 3 \alpha$

The first term on the right side of Eq. (8) represents the isotropic elastic energy, while the second term represents the core energy. In fact, since both $\mathbf{B} \boldsymbol{\alpha}$ and $\mathbf{B} \boldsymbol{\delta}$ are $30^{\circ}$ partials, $E_{\mathbf{B} \boldsymbol{\alpha}}=E_{\mathbf{B} \delta}$. This leads to $\Delta E_{E q 1}^{B \alpha \rightarrow B \delta+\delta \alpha}=E_{\delta \alpha}$. For easy comparison we will hereafter define

$\widehat{E}=\frac{G a^{2}}{72 \pi(1-v)} \ln \frac{\sqrt{2} d}{a}=\widetilde{E} \ln \frac{\sqrt{2} d}{a}$

Assuming hereafter that the Poisson's ratio is approximately $1 / 3$, which is a reasonable approximation for most fcc metals, one can easily calculate $\Delta E_{E q 1}^{B \alpha \rightarrow B \delta+\delta \alpha}=\widehat{E}+$ $2.0 \widetilde{E}$. Note that $\widehat{E}$ is much larger than $\widetilde{E}$. For example, assuming the grain size is $50 \mathrm{~nm}$ and $a$ is $0.4 \mathrm{~nm}$, we have $\widehat{E} \approx 5 \widetilde{E}$.

Following a similar procedure, the energy barrier for the dislocation reaction in Eq. (2) can be calculated as:

$$
\begin{aligned}
\Delta E_{E q 2}^{\delta \alpha \rightarrow \delta B+B \alpha} & \approx \frac{G a^{2}(10-9 v)}{144 \pi(1-v)} \ln \frac{\sqrt{2} d}{a}+5.5 \widetilde{E} \\
& \approx 3.5 \widehat{E}+5.5 \widetilde{E}
\end{aligned}
$$

This indicates that the second reaction requires a much higher applied stress to overcome the energy barrier. If the applied stress is high enough to activate the reaction described in Eq. (1) but not the reaction in Eq. (2), then a step will be produced at the twin boundary. Twin boundaries with steps have been extensively studied experimentally and by MD simulations [33,36,37]. For example, Yamakov et al. [36] show that a partial dislocation can interact with a twin boundary, which forms a stair-rod dislocation and a step at the twin boundary and, consequently, thickens or reduces the twin by one atomic layer. 


\subsection{Transmission of the $30^{\circ}$ partial across the twin boundary}

To understand how the $30^{\circ}$ partial $\mathbf{B} \boldsymbol{\alpha}$ on the BCD slip plane can be transmited across the twin boundary we need to invoke the double Thompson tetrahedron [28], illustrated in Fig. 4. As shown, the Thompson tetrahedron above the (1 1111$)$ twin boundary represents matrix slip systems, while the bottom tetrahedron represents twin slip systems. The twin boundary plane is shared by the matrix above it and the twin below it. Therefore, the matrix tetrahedron and the twin tetrahedron share the same base, which is $\mathrm{ABC}$. In other words, dislocations with Burgers vectors $\mathbf{A B}, \mathbf{B C}, \mathbf{C A}, \mathbf{A} \boldsymbol{\delta}, \mathbf{B} \boldsymbol{\delta}$, and $\mathbf{C} \boldsymbol{\delta}$ can slip both in the matrix and in the twin.

From Fig. 4 the $30^{\circ}$ partial $\mathbf{B} \boldsymbol{\alpha}$ on the BCD slip plane in the matrix, can have the following dislocation reaction to release another partial in the twin:

$\mathbf{B} \boldsymbol{\alpha} \rightarrow \mathbf{B} \boldsymbol{\alpha}^{\prime}+\boldsymbol{\alpha}^{\prime} \boldsymbol{\alpha}$

where $\mathbf{B} \boldsymbol{\alpha}^{\prime}$ is a partial that can slip away in the twin from the twin boundary on the $\mathrm{BCD}^{\prime}$ plane, and $\boldsymbol{\alpha}^{\prime} \boldsymbol{\alpha}$ is a new type of stationary stair-rod dislocation across the twin boundary. Since the dislocation line is parallel to BC, it can be seen from Fig. 4 that $\alpha^{\prime} \alpha$ is an edge dislocation with its Burgers vector perpendicular to both the dislocation line and the twin boundary ( $\left.\begin{array}{lll}1 & 1 & 1\end{array}\right)$. The magnitude of $\alpha^{\prime} \alpha$ can be calculated from the geometry of the double tetrahedron as $2 a / 3 \sqrt{ } 3$. The dislocation configuration after the reaction is illustrated in Fig. 5, which shows two stacking faults from the two sides of the twin boundary meeting at the twin boundary and connected by the stair-rod dislocation $\alpha^{\prime} \alpha$.

The energy barrier of the dislocation reaction described in Eq. (11) can be calculated as:

$$
\begin{aligned}
\Delta E_{E q 11}^{B \alpha \rightarrow B \alpha^{\prime}+\alpha^{\prime} \alpha} & \approx \frac{G a^{2}}{27 \pi(1-v)} \ln \frac{\sqrt{2} d}{a}+4.1 \widetilde{E} \\
& \approx 2.7 \widehat{E}+4.1 \widetilde{E}
\end{aligned}
$$

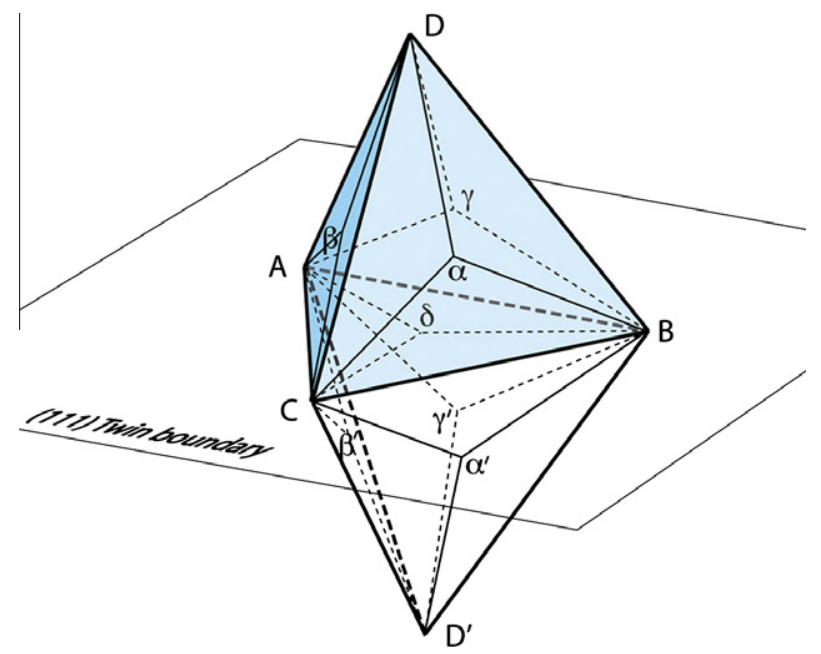

Fig. 4. Illustration of a double Thompson tetrahedron. The top tetrahedron above the $\left(\begin{array}{lll}1 & 1 & 1\end{array}\right)$ twin boundary represents matrix slip systems, while the bottom tetrahedron represents twin slip systems.

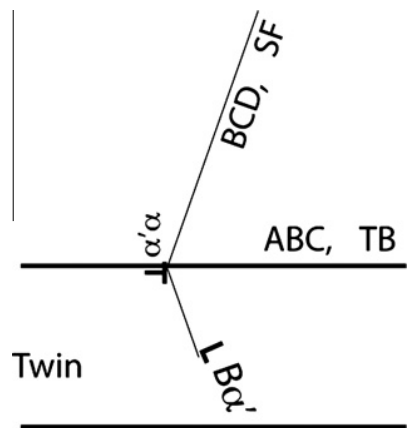

Fig. 5. The dislocation configuration after the partial $\mathrm{B} \alpha$ penetrates the twin boundary to release another partial $\mathrm{B} \alpha^{\prime}$ on the $\mathrm{BCD}^{\prime}$ plane inside the twin, leaving a stair-rod dislocation $\alpha^{\prime} \alpha$ on the twin boundary. This configuration is viewed from the $\mathrm{BC}$ orientation.

This indicates that the energy barrier for the $30^{\circ}$ partial $\mathbf{B} \boldsymbol{\alpha}$ to be transmited across the twin boundary is smaller than that in reaction 2, suggesting that this scenario is energetically plausible under an appropriate applied stress.

\section{A $90^{\circ}$ partial at the twin boundary}

Assuming that a $90^{\circ}$ Shockley partial dislocation $\mathbf{D} \boldsymbol{\alpha}$ glides on the BCD plane towards the coherent twin boundary, we describe plausible dislocation reactions at the twin boundary below.

\subsection{Cross-slip of the $90^{\circ}$ partial at the twin boundary}

The cross-slip of $90^{\circ}$ partials at the coherent twin boundary has been reported to be responsible for the formation of fivefold twins $[47,48]$, which have been experimentally observed and also verified by MD simulation [49]. Since this has been reported before, we will give only a brief description here.

A deformation twin can be defined by the twin plane $K_{1}$, shear direction $\eta_{1}$, undistorted plane $K_{2}$, and direction $\eta_{2}$ (see Fig. 6) [45]. Deformation twins in an fcc metal are of compound type, in which a twin formed by Shockley partials with Burgers vectors parallel to $\eta_{1}$ gliding on $K_{1}$ is the same as a twin formed by partials with Burgers vectors parallel to $\eta_{2}$ gliding on $K_{2}$. In the current situation $K_{1}$ and $K_{2}$ correspond to the $\mathrm{ABC}$ and $\mathrm{BCD}$ planes in the Thompsons tetrahedron, respectively. $\eta_{1}$ is parallel to the $90^{\circ}$ partial $\mathbf{A} \boldsymbol{\delta}$ and $\eta_{2}$ is parallel to another $90^{\circ}$ partial $\mathbf{D} \boldsymbol{\alpha}$. In other words, when the $90^{\circ}$ partial $\mathbf{D} \boldsymbol{\alpha}$ on the BCD plane reaches the twin boundary it becomes equivalent to another $90^{\circ}$ partial $\mathbf{A} \boldsymbol{\delta}$ on the ABC plane. Under an appropriate external shear stress the A $\boldsymbol{\delta}$ partial could glide to the left or right, which consequently grows or shrinks the twin by one atomic layer. Note that since both $\mathbf{D} \boldsymbol{\alpha}$ and $\mathbf{A} \boldsymbol{\delta}$ are the same type of partial, there is no energy change (barrier) in this dislocation reaction. Therefore, orientation of the applied stress becomes a critical factor influencing this cross-slip. 


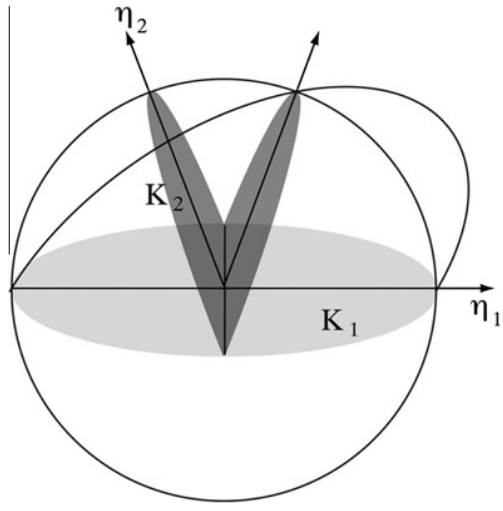

Fig. 6. Illustration of twinning elements $\eta_{1}, \eta_{2}, K_{1}$ and $K_{2}$.

\subsection{Transmission of the $90^{\circ}$ partial across the twin boundary}

To understand how a $90^{\circ}$ partial $\mathbf{D} \boldsymbol{\alpha}$ can be transmitted across the twin boundary to activate a partial dislocation in the twin we need to take a careful look at the double Thompson tetrahedron to determine the dislocation reactions that can translate the Burgers vector of the $90^{\circ}$ partial into a partial in the twin. As shown in Fig. 4, the $90^{\circ}$ partial $\mathbf{D} \boldsymbol{\alpha}$ can dissociate as:

$\mathbf{D} \boldsymbol{\alpha} \rightarrow \mathbf{D} \boldsymbol{\delta}+\boldsymbol{\delta} \boldsymbol{\alpha}$

The double Thompson tetrahedron indicates that the Burgers vector $\mathbf{D} \boldsymbol{\delta}$ is identical to $\delta \mathbf{D}^{\prime}$ in the twin, i.e. $\mathbf{D} \boldsymbol{\delta}=\delta \mathbf{D}^{\prime}$, which can dissociate into a $90^{\circ}$ partial $\boldsymbol{\alpha}^{\prime} \mathbf{D}^{\prime}$ and a stair-rod dislocation $\boldsymbol{\delta} \boldsymbol{\alpha}^{\prime}$, i.e.

$\mathbf{D} \boldsymbol{\delta}=\delta \mathbf{D}^{\prime} \rightarrow \boldsymbol{\delta} \boldsymbol{\alpha}^{\prime}+\boldsymbol{\alpha}^{\prime} \mathbf{D}^{\prime}$

The $90^{\circ}$ partial $\alpha^{\prime} \mathbf{D}^{\prime}$ can glide in the twin to move away from the twin boundary. Other similar reactions that can also produce a $90^{\circ}$ partial include:

$$
\delta \mathbf{D}^{\prime} \rightarrow \boldsymbol{\delta} \boldsymbol{\beta}^{\prime}+\boldsymbol{\beta}^{\prime} \mathbf{D}^{\prime}
$$

and

$\boldsymbol{\delta} \mathbf{D}^{\prime} \rightarrow \boldsymbol{\delta} \boldsymbol{\gamma}^{\prime}+\boldsymbol{\gamma}^{\prime} \mathbf{D}^{\prime}$

The $90^{\circ}$ partials $\boldsymbol{\beta}^{\prime} \mathbf{D}^{\prime}$ and $\gamma^{\prime} \mathbf{D}^{\prime}$ can glide on the $\mathrm{ACD}^{\prime}$ and $\mathrm{ABD}^{\prime}$ slip planes, respectively, in the twin.

The magnitude of $\mathbf{D} \boldsymbol{\delta}$ and $\boldsymbol{\delta} \mathbf{D}^{\prime}$ is $a / \sqrt{ } 3$ according to the double Thompson tetrahedron. The energy barrier of the dislocation reaction described in Eq. (13) can be calculated as:

$\Delta E_{E q 13}^{D \alpha \rightarrow D \delta+\delta \alpha} \approx \frac{G a^{2}}{18 \pi(1-v)} \ln \frac{\sqrt{2} d}{a}+4.4 \widetilde{E}=4 \widehat{E}+4.4 \widetilde{E}$

The energy barrier of the dislocation reaction described in Eq. (14) can be calculated as:

$\Delta E_{E q 14}^{\delta D^{\prime} \rightarrow \delta \alpha^{\prime}+\alpha^{\prime} D^{\prime}} \approx-\frac{G a^{2}}{36 \pi(1-v)} \ln \frac{\sqrt{2} d}{a}-0.3 \widetilde{E} \approx-2 \widehat{E}-0.3 \widetilde{E}$
The energy barriers for the reactions in Eqs. (15) and (16) are the same as that in Eq. (14). Therefore, the dislocation reaction described in Eq. (13) has a very high energy barrier while the reactions in Eqs. (14)-(16) are energetically favorable.

The high energy barrier in Eq. (13) is caused by creation of the dislocation $\mathbf{D} \boldsymbol{\delta}$ (or $\boldsymbol{\delta} \mathbf{D}^{\prime}$ ), which has a large Burgers vector with a magnitude of $a / \sqrt{ } 3$. If the dislocation reaction path changes to avoid the formation of $\mathbf{D} \boldsymbol{\delta}\left(\boldsymbol{\delta} \mathbf{D}^{\prime}\right)$, the energy barrier will be lower. Assuming that the dislocation reactions in Eqs. (13)-(15) take place in one step without forming $\mathbf{D} \boldsymbol{\delta}\left(\boldsymbol{\delta} \mathbf{D}^{\prime}\right)$, we can substitute Eq. (14) into Eq. (13), which yields:

$\mathbf{D} \boldsymbol{\alpha} \rightarrow \boldsymbol{\delta} \boldsymbol{\alpha}+\boldsymbol{\delta} \boldsymbol{\alpha}^{\prime}+\boldsymbol{\alpha}^{\prime} \mathbf{D}^{\prime}$

Similarly, $\boldsymbol{\beta}^{\prime} \mathbf{D}^{\prime}$ and $\gamma^{\prime} \mathbf{D}^{\prime}$ can also be formed by the following reactions:

$\mathbf{D} \boldsymbol{\alpha} \rightarrow \boldsymbol{\delta} \boldsymbol{\alpha}+\boldsymbol{\delta} \boldsymbol{\beta}^{\prime}+\boldsymbol{\beta}^{\prime} \mathbf{D}^{\prime}$

and

$\mathbf{D} \boldsymbol{\alpha} \rightarrow \boldsymbol{\delta} \boldsymbol{\alpha}+\boldsymbol{\delta} \boldsymbol{\gamma}^{\prime}+\boldsymbol{\gamma}^{\prime} \mathbf{D}^{\prime}$

The energy barrier for the reaction in Eq. (19) can be calculated as:

$\Delta E_{E q 19}^{D \alpha \rightarrow \delta \alpha+\delta \alpha^{\prime}+\alpha^{\prime} D^{\prime}} \approx \frac{G a^{2}}{36 \pi(1-v)} \ln \frac{\sqrt{2} d}{a}+4.0 \widetilde{E} \approx 2.0 \widehat{E}+4.0 \widetilde{E}$

Therefore, the energy barrier for the reaction in Eq. (19) is half of the energy barrier in Eq. (13). The energy barriers for the reactions in Eqs. (20) and (21) are identical to that in Eq. (19).

There are two stair-rod dislocations in Eqs. (19)-(21). Both stair-rod dislocations remain at the twin boundary and could react to form a dislocation structure with lower energy. With the help of the double Thompson tetrahedron the stair-rod reactions can be described by:

$$
\begin{aligned}
& \boldsymbol{\delta} \boldsymbol{\alpha}+\boldsymbol{\delta} \boldsymbol{\alpha}^{\prime} \rightarrow 4 / 9 \mathbf{A} \boldsymbol{\delta} \\
& \boldsymbol{\delta} \boldsymbol{\alpha}+\boldsymbol{\delta} \boldsymbol{\beta}^{\prime} \rightarrow 2 / 9 \boldsymbol{\delta} \mathbf{C} \\
& \boldsymbol{\delta} \boldsymbol{\alpha}+\boldsymbol{\delta} \boldsymbol{\gamma}^{\prime} \rightarrow 2 / 9 \boldsymbol{\delta} \mathbf{B}
\end{aligned}
$$

Substituting Eqs. (23)-(25) into Eqs. (19)-(21) yields:

$$
\begin{aligned}
& \mathbf{D} \boldsymbol{\alpha} \rightarrow 4 / 9 \mathbf{A} \boldsymbol{\delta}+\boldsymbol{\alpha}^{\prime} \mathbf{D}^{\prime} \\
& \mathbf{D} \boldsymbol{\alpha} \rightarrow 2 / 9 \boldsymbol{\delta} \mathbf{C}+\boldsymbol{\beta}^{\prime} \mathbf{D}^{\prime}
\end{aligned}
$$

and

$\mathbf{D} \boldsymbol{\alpha} \rightarrow 2 / 9 \delta \mathbf{B}+\gamma^{\prime} \mathbf{D}^{\prime}$

The energy barrier for reaction in Eq. (26) is:

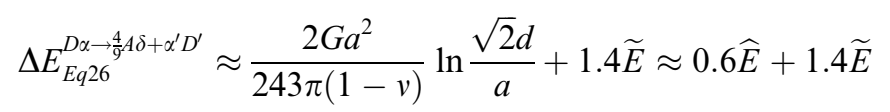

The energy barriers for the reactions in Eqs. (27) and (28) are: 
$\Delta E_{E q 27,28} \approx \frac{2 G a^{2}(1-3 v / 4)}{486 \pi(1-v)} \ln \frac{\sqrt{2} d}{a}+0.2 \widetilde{E} \approx 0.1 \widehat{E}+0.2 \widetilde{E}$

Therefore, the energy barriers for dislocation reactions in Eqs. (26)-(28) are very low, which makes it easier for the $90^{\circ}$ partial $\mathbf{D} \boldsymbol{\alpha}$ to be transmitted across the twin boundary to emit another $90^{\circ}$ partial in the twin. However, it is also noted that the reaction products in these equations include dislocations with Burgers vectors that are a fraction of that of a partial, which may make the energy higher than described in Eqs. (29) and (30), since they do not correspond to stable or metastable atomic positions.

\section{Reaction of a perfect screw dislocation at the twin boundary}

If we assume that a perfect screw dislocation $\mathbf{B C}$ glides on the BCD plane toward the twin boundary (see Fig. 4) it could be dissociated into two $30^{\circ}$ partials with a stacking fault in between, i.e.

$\mathrm{BC} \rightarrow \mathbf{B} \boldsymbol{\alpha}+\boldsymbol{\alpha C}$

when this dissociated $\mathbf{B C}$ reaches the twin boundary it could constrict to again form a perfect dislocation. Since $\mathbf{B C}$ is parallel to the dislocation line, it can either cross-slip into the $\mathrm{ABC}$ plane on the twin boundary or onto the $\mathrm{BCD}^{\prime}$ plane in the twin, depending on the orientation of the applied stress. Therefore, a screw dislocation can easily cross-slip on the twin boundary or be transmitted across the twin boundary. The interaction of a screw dislocation with a coherent twin boundary has been observed by MD simulation [34].

\section{Reaction of a perfect $60^{\circ}$ dislocation at the twin boundary}

If we assume that a perfect $60^{\circ}$ dislocation BD glides on the BCD plane towards the twin boundary (see Fig. 4) it could be dissociated into a $30^{\circ}$ partial $\mathbf{B} \boldsymbol{\alpha}$ and a $90^{\circ}$ partial $\alpha \mathrm{D}$ with a stacking fault between them, i.e. $\mathbf{B D} \rightarrow \mathbf{B} \boldsymbol{\alpha}+$ $\boldsymbol{\alpha D}$. These partials $(\mathbf{B} \boldsymbol{\alpha}$ and $\boldsymbol{\alpha D})$ with a stacking fault ribbon can glide together under an applied stress towards the twin boundary. Since the perfect $60^{\circ}$ dislocation cannot easily cross-slip or be transmitted across the twin boundary, there are several plausible scenarios that could occur, which are described below.

\subsection{Perfect dislocation BD constricts before reaction}

We begin by describing the scenario where the partials are constricted to form the perfect dislocation BD before the dislocation reacts at the twin boundary. Such a scenario can happen more easily when the stacking fault energy is relatively high and the distance between the leading and trailing partials is small. This has been observed experimentally [32] and by MD simulations $[33,35,50]$. It can be seen from the double Thompson tetrahedron (Fig. 4) that BD is at a $60^{\circ}$ angle to the dislocation line $\mathrm{BC}$. The dislocation reaction for $\mathrm{BD}$ to cross-slip onto the $\mathrm{ABC}$ plane is:

$\mathrm{BD} \rightarrow \mathrm{BC}+\mathrm{CD}$

The energy barrier for such a reaction is:

$\Delta E_{E q 32}^{B D \rightarrow B C+C D} \approx \frac{G a^{2}}{8 \pi} \ln \frac{\sqrt{2} d}{a}+7.2 \widetilde{E} \approx 6 \widehat{E}+7.2 \widetilde{E}$

This energy barrier is so high that such a cross-slip is almost impossible.

Another possible dislocation reaction is for BD to be transmitted across the twin boundary to emit a perfect dislocation in the twin. The following analysis yields possible dislocation reactions. First, BD can dissociate according to: $\mathrm{BD} \rightarrow \mathbf{B} \boldsymbol{\delta}+\boldsymbol{\delta} \mathbf{D}$

where $\boldsymbol{\delta} \mathbf{D}$ is equivalent to $\mathbf{D}^{\prime} \boldsymbol{\delta}$, which can further react to emit perfect dislocations in the twin according to:

$\begin{aligned} \boldsymbol{\delta} \mathbf{D} & =\mathbf{D}^{\prime} \boldsymbol{\delta} \rightarrow \mathbf{D}^{\prime} \mathbf{B}+\mathbf{B} \boldsymbol{\delta} \\ \boldsymbol{\delta} \mathbf{D} & =\mathbf{D}^{\prime} \boldsymbol{\delta} \rightarrow \mathbf{D}^{\prime} \mathbf{A}+\mathbf{A} \boldsymbol{\delta} \\ \boldsymbol{\delta} \mathbf{D} & =\mathbf{D}^{\prime} \boldsymbol{\delta} \rightarrow \mathbf{D}^{\prime} \mathbf{C}+\mathbf{C} \boldsymbol{\delta}\end{aligned}$

Substituting Eqs. (35)-(37) into Eq. (34), and also considering $\mathbf{B} \boldsymbol{\delta}+\mathbf{A} \boldsymbol{\delta}=\boldsymbol{\delta} \mathbf{C}$ and $\mathbf{B} \boldsymbol{\delta}+\mathbf{C} \boldsymbol{\delta}=\boldsymbol{\delta} \mathbf{A}$, we have:

$\mathbf{B D} \rightarrow 2 \mathbf{B} \boldsymbol{\delta}+\mathbf{D}^{\prime} \mathbf{B}$

$\mathrm{BD} \rightarrow \boldsymbol{\delta} \mathbf{C}+\mathbf{D}^{\prime} \mathbf{A}$

$\mathrm{BD} \rightarrow \boldsymbol{\delta} \mathbf{A}+\mathbf{D}^{\prime} \mathbf{C}$

The energy barriers for the dislocation reactions in Eqs. (38)-(40) can be described by:

$$
\begin{aligned}
\Delta E_{E q 38}^{B D \rightarrow 2 B \delta+D^{\prime} B} & \approx \frac{G a^{2}(1-3 v / 4)}{12 \pi(1-v)} \ln \frac{\sqrt{2} d}{a}+7.5 \widetilde{E} \\
& \approx 4.5 \widehat{E}+7.5 \widetilde{E} \\
\Delta E_{E q 39}^{B D \rightarrow \delta C+D^{\prime} A} & \approx \frac{G a^{2}}{24 \pi(1-v)} \ln \frac{\sqrt{2} d}{a}+3.9 \widetilde{E} \\
& \approx 3.0 \widehat{E}+3.9 \widetilde{E} \\
\Delta E_{E q 40}^{B D \rightarrow \delta A+D^{\prime} C} & \approx \frac{G a^{2}}{24 \pi(1-v)} \ln \frac{\sqrt{2} d}{a}+3.9 \widetilde{E} \\
& \approx 3.9 \widehat{E}+3.9 \widetilde{E}
\end{aligned}
$$

Therefore, the dislocation reactions in Eqs. (39) and (40) have smaller energy barriers. In these two reactions one partial will glide on the twin plane, which will grow or shrink the twin by one atomic plane and leave behind a step on the twin boundary depending on the gliding direction. At the same time a perfect $60^{\circ}$ dislocation is emitted in the twin, which will also produce a step on the twin boundary. In comparison, the dislocation reaction in Eq. (38) will release two partials on the $\mathrm{ABC}$ plane, which may grow or shrink the twin by two atomic planes if they glide on two slip planes.

\section{2. $30^{\circ}$ leading partial $\boldsymbol{B} \boldsymbol{\alpha}$ reacts first at the twin boundary}

If we assume that the $60^{\circ}$ dislocation $\mathbf{B D}$ glides on the BCD plane toward the twin boundary it can be dissociated 
as $\mathbf{B D} \rightarrow \mathbf{B} \boldsymbol{\alpha}+\boldsymbol{\alpha D}$, with the $30^{\circ}$ partial $\mathbf{B} \boldsymbol{\alpha}$ as the leading partial. The leading partial $\mathbf{B} \boldsymbol{\alpha}$ could either cross-slip onto the twin boundary plane or be transmitted across the twin boundary. We will discuss these two cases below.

\subsubsection{Leading $30^{\circ}$ partial $B \alpha$ cross-slip onto the twin boundary plane}

When the stacking fault energy is relatively low and under an appropriate applied stress $\mathbf{B} \boldsymbol{\alpha}$ can cross-slip onto the $\mathrm{ABC}$ twin boundary plane to emit a partial $\mathbf{B} \boldsymbol{\delta}$ following the dislocation reaction described in Eq. (1), leaving behind a stair-rod dislocation $\delta \boldsymbol{\alpha}$ and the $90^{\circ}$ partial $\alpha \mathbf{D}$, still on the BCD plane, as shown in Fig. 7a. Such a dislocation structure has been observed both experimentally $[3,51]$ and by MD simulation [9,33,37], and it has been assumed to be very effective in blocking other dislocations and, consequently, causing strain hardening [9].

As shown in Fig. 7a, under a high applied stress the trailing partial $\alpha \mathbf{D}$ may be driven to the twin boundary to react with the stair-rod dislocation $\delta \boldsymbol{\alpha}$ :

$\delta \alpha+\alpha \mathbf{D} \rightarrow \delta \mathbf{D}$

The energy barrier for this reaction is:

$$
\begin{aligned}
\Delta E_{E q 44}^{\delta \alpha+\alpha D \rightarrow \delta D} & \approx-\frac{G a^{2}}{36 \pi(1-v)} \ln \frac{\sqrt{2} d}{a}+0.3 \widetilde{E} \\
& \approx-2 \widehat{E}+0.3 \widetilde{E}
\end{aligned}
$$

Therefore, the dislocation reaction in Eq. (44) is energetically favorable.

From the double Thompson tetrahedron $\delta \mathbf{D}$ is equivalent to $\mathbf{D}^{\prime} \boldsymbol{\delta}$. The latter can dissociate as:

$\mathbf{D}^{\prime} \boldsymbol{\delta} \rightarrow \mathbf{D}^{\prime} \boldsymbol{\alpha}^{\prime}+\boldsymbol{\alpha}^{\prime} \boldsymbol{\delta}$

where $\mathbf{D}^{\prime} \boldsymbol{\alpha}^{\prime}$ can slip away on the $\mathrm{BCD}^{\prime}$ plane in the twin. This scenario is schematically illustrated in Fig. 7b. The energy barrier for this reaction is:

$$
\begin{aligned}
\Delta E_{E q 46}^{D^{\prime} \rightarrow D^{\prime} \alpha^{\prime}+\alpha^{\prime} \delta} & \approx \frac{G a^{2}}{36 \pi(1-v)} \ln \frac{\sqrt{2} d}{a}-0.3 \widetilde{E} \\
& \approx 2 \widehat{E}-0.3 \widetilde{E}
\end{aligned}
$$

This energy barrier is reasonably low for it to occur during real deformation.

Another scenario is for the stair-rod dislocation $\delta \boldsymbol{\alpha}$ in Fig. 7a to further dissociate into two partial dislocations according to Eq. (2), $\delta \boldsymbol{\alpha} \rightarrow \delta \mathbf{B}+\mathbf{B} \boldsymbol{\alpha}$, where the partial $\boldsymbol{\delta} \mathbf{B}$ glides on the twin boundary in the opposite direction to $\mathbf{B} \boldsymbol{\delta}$ to reduce the twin thickness by one atomic plane, as illustrated in Fig. 7c. Such a process can be repeated to reduce the twin, as described in Section 3.1. The energy barrier for such a reaction is about $3.5 \widehat{E}+5.5 \widetilde{E}$, making it more difficult than the reaction in Eq. (46). The cutting of a stacking fault by a dislocation has been observed by MD simulation [36]. The de-twinning process is similar to cutting of the stacking fault, suggesting that such a scenario could happen under an appropriate applied stress.

\subsubsection{Leading $30^{\circ}$ partial $B \alpha$ transmits across the twin boundary}

As discussed in Section 3.2 (see Eq. (11) and Fig. 5), the $30^{\circ}$ partial $\mathbf{B} \boldsymbol{\alpha}$ may be transmited across the twin boundary, leaving behind a large stair-rod dislocation $\alpha \alpha^{\prime}$. If the trailing partial $\alpha \mathbf{D}$ remains on the BCD plane it will have a unique dislocation configuration where one stacking fault links $\boldsymbol{\alpha} \boldsymbol{\alpha}^{\prime}$ with partial $\mathbf{B} \boldsymbol{\alpha}^{\prime}$, while another stacking fault links $\boldsymbol{\alpha} \boldsymbol{\alpha}^{\prime}$ with trailing partial $\boldsymbol{\alpha D}$, as shown in Fig. 8a. The trailing partial $\alpha \mathbf{D}$ can also cross-slip onto the twin boundary plane, becoming a partial $\boldsymbol{\delta} \mathbf{A}$ that slips to the left to increase the twin by one atomic plane (as described in Section 4.1), forming a dislocation configuration, as shown in Fig. $8 b$.

The trailing partial $\boldsymbol{\alpha D}$ can also react with the stair-rod $\alpha \alpha^{\prime}$ and be transmitted across the twin boundary considering $\alpha \mathbf{D} \rightarrow \delta \mathbf{D}+\boldsymbol{\alpha} \boldsymbol{\delta}$ and $\boldsymbol{\alpha} \boldsymbol{\alpha}^{\prime} \rightarrow \boldsymbol{\delta} \boldsymbol{\alpha}+\boldsymbol{\alpha}^{\prime} \boldsymbol{\delta}$ :

$\alpha \mathbf{D}+\alpha \alpha^{\prime} \rightarrow \delta \mathbf{D}+\alpha^{\prime} \boldsymbol{\delta}$

From the double Thompson tetrahedron (Fig. 4) it can be seen that $\boldsymbol{\delta} \mathbf{D}=\mathbf{D}^{\prime} \boldsymbol{\delta}=\boldsymbol{\alpha}^{\prime} \boldsymbol{\delta}+\mathbf{D}^{\prime} \boldsymbol{\alpha}^{\prime}$. Therefore, Eq. (48) can be rewritten as:

$\mathbf{D} \boldsymbol{\alpha}+\boldsymbol{\alpha} \boldsymbol{\alpha}^{\prime} \rightarrow 2 \boldsymbol{\alpha}^{\prime} \boldsymbol{\delta}+\mathbf{D}^{\prime} \boldsymbol{\alpha}^{\prime}$

The energy barrier for the reaction in Eq. (49) can be described by:

$\Delta E_{E q^{4}+\alpha \alpha^{\prime} \rightarrow 2 \alpha^{\prime} \delta+D^{\prime} \alpha^{\prime}}^{D} \approx \frac{G a^{2}}{18 \pi(1-v)} \ln \frac{\sqrt{2} d}{a}=4 \widehat{E}$

The dislocation reaction in Eq. (49) can release one partial $\left(\mathbf{D}^{\prime} \boldsymbol{\alpha}^{\prime}\right)$ in the twin, leaving behind a double stair-rod dislocation $\left(\mathbf{2} \boldsymbol{\delta} \boldsymbol{\alpha}^{\prime}\right)$. However, this reaction apparently has a high energy barrier.

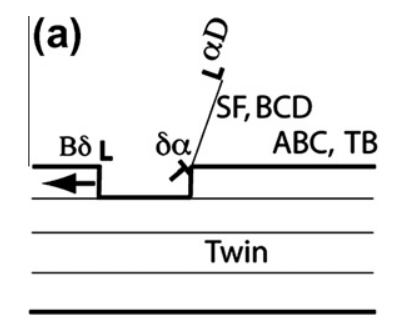

(b)

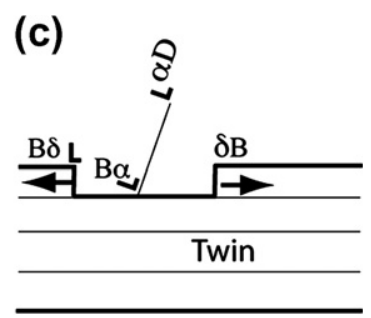

Fig. 7. Reaction of dissociated $60^{\circ}$ dislocation $\mathrm{BD}$ with a $30^{\circ}$ leading partial at the twin boundary. (a) The leading partial cross-slips onto the ABC twin boundary, leaving behind a stair-rod dislocation and the $90^{\circ}$ trailing partial still on the original BCD slip plane. (b) After the trailing partial $\alpha \mathrm{D}$ reacts with the stair-rod dislocation $\delta \alpha$ and transmits across the twin boundary (Eqs. (44) and (46)). (c) The stair-rod dislocation dissociates according to $\delta \alpha \rightarrow \delta \mathrm{B}+\mathrm{B} \alpha$ to reduce the twin by one atomic layer. 

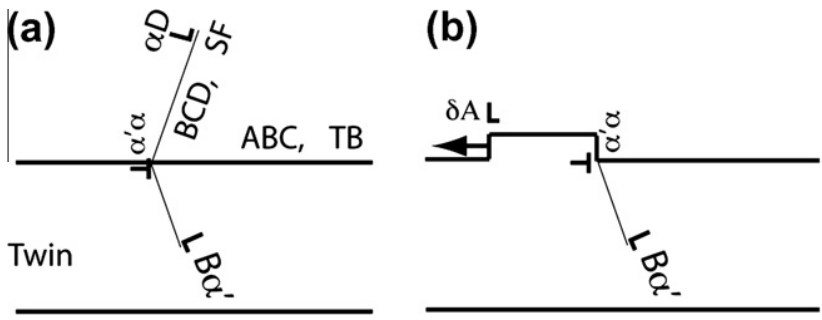

Fig. 8. (a) Dislocation configuration after the leading $30^{\circ}$ partial $\mathrm{B} \alpha$ transmits across the twin boundary and the trailing $90^{\circ}$ partial $\alpha \mathrm{D}$ remains on the BCD plane. (b) After the trailing partial $\alpha \mathrm{D}$ cross-slips into the $\mathrm{ABC}$ plane to grow the twin by one atomic plane.

Similarly to Eqs. (36) and (37), $\boldsymbol{\delta} \mathbf{D}$ could also dissociate according to the two equations: $\delta \mathbf{D}=\mathbf{D}^{\prime} \boldsymbol{\delta}=\mathbf{D} \boldsymbol{\beta}^{\prime}+\beta^{\prime} \boldsymbol{\delta}$ and $\boldsymbol{\delta} \mathbf{D}=\mathbf{D}^{\prime} \boldsymbol{\delta}=\mathbf{D} \boldsymbol{\gamma}^{\prime}+\boldsymbol{\gamma}^{\prime} \boldsymbol{\delta}$. However, the final energy barrier for such reactions will be even higher than that of the reaction in Eq. (49). Therefore, these reactions will not be described in detail here.

\section{3. $90^{\circ}$ leading partial $\alpha \boldsymbol{D}$ reacts first at the twin boundary}

If the $90^{\circ}$ leading partial $\alpha \mathbf{D}$ is the leading partial it could either cross-slip onto the twin boundary plane or transmit across the twin boundary. We will discuss these two cases below.

\subsubsection{Leading $90^{\circ}$ partial $\boldsymbol{\alpha D}$ cross-slips onto the twin boundary plane}

The leading partial $\boldsymbol{\alpha D}$ can cross-slip to form $\boldsymbol{\delta} \mathbf{A}$, which slips on the twin boundary plane ABC as described in Section 4.1. The trailing partial $\mathbf{B} \boldsymbol{\alpha}$ can also cross-slip onto the twin boundary to form $\mathbf{B} \boldsymbol{\delta}$ on the $\mathrm{ABC}$ plane, following the procedure described in Section 3.1, leaving behind a stairrod dislocation $\boldsymbol{\delta} \boldsymbol{\alpha} . \mathbf{B} \boldsymbol{\delta}$ and $\boldsymbol{\delta} \mathbf{A}$ can glide together on the twin boundary with a stacking fault between them.

On the other hand, the trailing $30^{\circ}$ partial $\mathbf{B} \alpha$ can also be transmited across the twin boundary to emit a partial $\mathbf{B} \boldsymbol{\alpha}^{\prime}$ in the twin, as described in Section 3.2 and Fig. 5. This leaves behind a stair-rod $\alpha^{\prime} \alpha$ at the twin boundary.

\subsubsection{Leading $90^{\circ}$ partial $\alpha D$ transmits across the twin boundary}

The leading partial $\boldsymbol{\alpha D}$ can be transmitted across the twin boundary, which is energetically not too difficult, as described in Section 4.2. With the help of Eqs. (19)-(21) in Section 4.2, which describe the dislocation reactions of $\mathbf{D} \boldsymbol{\alpha}$, the $\boldsymbol{\alpha D}$ reactions for transmission across the twin boundary can be described by:

$$
\begin{aligned}
& \alpha \mathbf{D} \rightarrow \alpha \boldsymbol{\delta}+\alpha^{\prime} \boldsymbol{\delta}+\mathbf{D}^{\prime} \alpha^{\prime} \\
& \alpha \mathbf{D} \rightarrow \alpha \boldsymbol{\delta}+\boldsymbol{\beta}^{\prime} \boldsymbol{\delta}+\mathbf{D}^{\prime} \boldsymbol{\beta}^{\prime}
\end{aligned}
$$

and

$$
\alpha \mathbf{D} \rightarrow \boldsymbol{\alpha} \boldsymbol{\delta}+\boldsymbol{\gamma}^{\prime} \boldsymbol{\delta}+\mathbf{D}^{\prime} \boldsymbol{\gamma}^{\prime}
$$

where the partial dislocations $\mathbf{D} \boldsymbol{\alpha}^{\prime}, \mathbf{D}^{\prime} \boldsymbol{\beta}^{\prime}$ or $\mathbf{D}^{\prime} \boldsymbol{\gamma}^{\prime}$ will glide away in the twin, leaving behind two stair-rod dislocations at the twin boundary. When the trailing partial $\mathbf{B} \boldsymbol{\alpha}$ reaches the twin boundary it can react with the stair-rod dislocation in all three cases, i.e.

$\mathrm{B} \alpha+\alpha \delta \rightarrow B \delta$

This reaction is energetically favorable with an energy reduction of $-\widehat{E}-2.0 \widetilde{E}$. The $\mathbf{B} \boldsymbol{\delta}$ can glide on the $\mathrm{ABC}$ plane, which either increases or reduces the twin thickness by one atomic plane, depending on the slip direction. The slip direction is determined by the orientation of the applied shear stress.

The trailing partial $\mathbf{B} \boldsymbol{\alpha}$ can also react with stair-rod dislocations (as shown in Eqs. (51)-(53)) at the twin boundary to release a Shockley partial dislocation in the twin:

$\mathbf{B} \boldsymbol{\alpha}+\boldsymbol{\alpha} \boldsymbol{\delta}+\boldsymbol{\alpha}^{\prime} \boldsymbol{\delta} \rightarrow \mathbf{B} \boldsymbol{\alpha}^{\prime}+2 \boldsymbol{\alpha}^{\prime} \boldsymbol{\delta}$
$\mathbf{B} \boldsymbol{\alpha}+\boldsymbol{\alpha} \boldsymbol{\delta}+\boldsymbol{\beta}^{\prime} \boldsymbol{\delta} \rightarrow \mathbf{B} \boldsymbol{\alpha}^{\prime}+\boldsymbol{\alpha}^{\prime} \boldsymbol{\delta}+\boldsymbol{\beta}^{\prime} \boldsymbol{\delta}$

and

$\mathbf{B} \boldsymbol{\alpha}+\boldsymbol{\alpha} \boldsymbol{\delta}+\boldsymbol{\gamma}^{\prime} \boldsymbol{\delta} \rightarrow \mathbf{B} \boldsymbol{\alpha}^{\prime}+\boldsymbol{\alpha}^{\prime} \boldsymbol{\delta}+\boldsymbol{\gamma}^{\prime} \boldsymbol{\delta}$

At first sight the energy barrier should be 0 for the reactions in Eqs. (55)-(57) because the number of partial dislocations and stair-rod dislocations do not change. However, as described in Eqs. (23)-(25), the reacting stair-rod dislocation pairs on the left can combine to form a dislocation with lower energy, while the stair-rod dislocation pairs on the right form a dislocation with higher energy when they combine together. These energy differences can be regarded as the energy barrier, which are described by:

$$
\begin{aligned}
& \Delta E_{E q 55} \approx \frac{23 G a^{2}}{486(1-v)} \ln \frac{\sqrt{2} d}{a}+2.7 \widetilde{E} \approx 3.4 \widehat{E}+2.7 \widetilde{E} \\
& \Delta E_{E q 56, E q 57} \approx 2.8 \widehat{E}+3.7 \widetilde{E}
\end{aligned}
$$

Therefore, the energy barrier for the trailing $30^{\circ}$ partial to be transmitted across the twin boundary is very high.

\section{Discussion}

Plausible dislocation reactions at the twin boundaries and the energy barriers associated with the reactions are listed in Table 1. It should be noted that the energy barrier is not the only factor that determines a dislocation reaction. Other factors that may play a role in the dislocation reaction include the orientation and magnitude of the applied stress, as well as the stacking fault energy of the material. If the grains are nearly randomly oriented without much texture it can be assumed that the orientation of the applied stress does not affect the global probability of which types of dislocation reaction occur.

Stacking fault energy should play a significant role in determining which types of dislocation reaction predominantly occur. Specifically, if the stacking fault energy is relatively high it will be difficult for partial dislocations to nucleate and slip, which leads to fewer interactions 
Table 1

Summary of dislocation reactions at twin boundaries and their isotropic energy barriers. TB = twin boundary, which is the ABC plane.

\begin{tabular}{|c|c|c|c|}
\hline Reaction description & Equation & Eq.\# & Isotropic energy barrier \\
\hline \multicolumn{4}{|l|}{$30^{\circ}$ Partial, $\boldsymbol{B} \boldsymbol{\alpha}$} \\
\hline Cross-slip onto the TB & $\mathbf{B} \boldsymbol{\alpha} \rightarrow \mathbf{B} \boldsymbol{\delta}+\boldsymbol{\delta} \boldsymbol{\alpha}$ & 1 & $\widehat{E}+2.0 \widetilde{E}$ \\
\hline Stair-rod dislocation dissociation & $\delta \boldsymbol{\alpha} \rightarrow \delta \mathbf{B}+\mathbf{B} \boldsymbol{\alpha}$ & 2 & $3.5 \widehat{E}+5.5 \widetilde{E}$ \\
\hline Transmit across the TB & $\mathbf{B} \boldsymbol{\alpha} \rightarrow \mathbf{B} \boldsymbol{\alpha}^{\prime}+\boldsymbol{\alpha}^{\prime} \boldsymbol{\alpha}$ & 11 & $2.7 \widehat{E}+4.1 \widetilde{E}$ \\
\hline \multicolumn{4}{|l|}{$90^{\circ}$ Partial, $\boldsymbol{D} \alpha$} \\
\hline Cross-slip onto the TB & $\mathbf{D} \boldsymbol{\alpha} \rightarrow \mathbf{A} \boldsymbol{\delta}$ & & 0 \\
\hline \multirow[t]{6}{*}{ Transmit across the TB } & $\mathbf{D} \boldsymbol{\alpha} \rightarrow \boldsymbol{\delta} \boldsymbol{\alpha}+\delta \boldsymbol{\alpha}^{\prime}+\boldsymbol{\alpha}^{\prime} \mathbf{D}^{\prime}$ & 19 & $2.0 \widehat{E}+4.0 \widetilde{E}$ \\
\hline & $\mathbf{D} \boldsymbol{\alpha} \rightarrow \boldsymbol{\delta} \boldsymbol{\alpha}+\boldsymbol{\delta} \boldsymbol{\beta}^{\prime}+\boldsymbol{\beta}^{\prime} \mathbf{D}^{\prime}$ & 20 & $2.0 \widehat{E}+4.0 \underset{\sim}{\widetilde{E}}$ \\
\hline & $\mathbf{D} \boldsymbol{\alpha} \rightarrow \boldsymbol{\delta} \boldsymbol{\alpha}+\boldsymbol{\delta} \boldsymbol{\gamma}^{\prime}+\boldsymbol{\gamma}^{\prime} \mathbf{D}^{\prime}$ & 21 & $2.0 \widehat{\underline{E}}+4.0 \underset{\sim}{\widetilde{E}}$ \\
\hline & $\mathbf{D} \boldsymbol{\alpha} \rightarrow 4 / 9 \mathbf{A} \boldsymbol{\delta}+\boldsymbol{\alpha}^{\prime} \mathbf{D}^{\prime}$ & 26 & 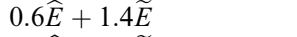 \\
\hline & $\mathbf{D} \boldsymbol{\alpha} \rightarrow 2 / 9 \delta \mathbf{B C}+\boldsymbol{\beta}^{\prime} \mathbf{D}^{\prime}$ & 27 & $0.1 \widehat{E}+0.2 \widetilde{\sim}$ \\
\hline & $\mathbf{D} \boldsymbol{\alpha} \rightarrow 2 / 9 \boldsymbol{\delta}+\boldsymbol{\gamma}^{\prime} \mathbf{D}^{\prime}$ & 28 & $0.1 \widehat{E}+0.2 \widetilde{E}$ \\
\hline \multicolumn{4}{|l|}{ Cross-slip of perfect screw dislocation, $\boldsymbol{B C}$} \\
\hline No dislocation reaction needed & & & 0 \\
\hline \multicolumn{4}{|l|}{ Perfect $60^{\circ}$ dislocation } \\
\hline Cross-slip onto TB & $\mathbf{B D} \rightarrow \mathbf{B C}+\mathbf{C D}$ & 32 & $6.0 \widehat{E}+7.2 \underset{\sim}{\widetilde{E}}$ \\
\hline \multirow[t]{3}{*}{ Transmit across the TB } & $\mathbf{B D} \rightarrow 2 \mathbf{B} \boldsymbol{\delta}+\mathbf{D}^{\prime} \mathbf{B}$ & 38 & $4.5 \widehat{E}+7.5 \widetilde{E}$ \\
\hline & $\mathbf{B D} \rightarrow \boldsymbol{\delta} \mathbf{C}+\mathbf{D}^{\prime} \mathbf{A}$ & 39 & $3.0 \widehat{\underline{E}}+3.9 \underset{\sim}{\widetilde{E}}$ \\
\hline & $\mathbf{B D} \rightarrow \boldsymbol{\delta} \mathbf{A}+\mathbf{D}^{\prime} \mathbf{C}$ & 40 & $3.0 \widehat{E}+3.9 \widetilde{E}$ \\
\hline $\begin{array}{l}30^{\circ} \text { Leading partial cross-slip onto } \mathrm{TB} \text { and trailing } \\
90^{\circ} \text { partial transmit across } \mathrm{TB}\end{array}$ & $\mathbf{D}^{\prime} \boldsymbol{\delta} \rightarrow \mathbf{D}^{\prime} \boldsymbol{\alpha}^{\prime}+\boldsymbol{\alpha}^{\prime} \boldsymbol{\delta}$ & 46 & $2.0 \widehat{E}-0.3 \widetilde{E}$ \\
\hline $\begin{array}{l}30^{\circ} \text { Leading partial transmit across TB first and the } \\
90^{\circ} \text { trailing partial transmit cross TB second }\end{array}$ & $\mathbf{D} \boldsymbol{\alpha}+\boldsymbol{\alpha} \boldsymbol{\alpha}^{\prime} \rightarrow 2 \boldsymbol{\alpha}^{\prime} \boldsymbol{\delta}+\mathbf{D}^{\prime} \boldsymbol{\alpha}^{\prime}$ & 49 & $4.0 \widehat{E}$ \\
\hline \multirow{3}{*}{$\begin{array}{l}90^{\circ} \text { Leading partial and } 30^{\circ} \text { trailing partial } \\
\text { transmit across } \mathrm{TB} \text { sequentially }\end{array}$} & $\mathbf{B} \boldsymbol{\alpha}+\boldsymbol{\alpha} \boldsymbol{\delta}+\boldsymbol{\alpha}^{\prime} \boldsymbol{\delta} \rightarrow \mathbf{B} \boldsymbol{\alpha}^{\prime}+2 \boldsymbol{\alpha}^{\prime} \boldsymbol{\delta}$ & 55 & $3.4 \widehat{E}+2.7 \widetilde{E}$ \\
\hline & $\mathbf{B} \boldsymbol{\alpha}+\boldsymbol{\alpha} \boldsymbol{\delta}+\boldsymbol{\beta}^{\prime} \boldsymbol{\delta} \rightarrow \mathbf{B} \boldsymbol{\alpha}_{\prime}+\boldsymbol{\alpha}^{\prime} \boldsymbol{\delta}+\boldsymbol{\beta}^{\prime} \boldsymbol{\delta}$ & 56 & $2.8 \widehat{E}+3.7 \widetilde{E}$ \\
\hline & $\mathbf{B} \boldsymbol{\alpha}+\boldsymbol{\alpha} \boldsymbol{\delta}+\gamma^{\prime} \boldsymbol{\delta} \rightarrow \mathbf{B} \boldsymbol{\alpha}^{\prime}+\boldsymbol{\alpha}^{\prime} \boldsymbol{\delta}+\gamma^{\prime} \boldsymbol{\delta}$ & 57 & $2.8 \widehat{E}+3.7 \widetilde{E}$ \\
\hline
\end{tabular}

$* \widehat{E}=\frac{G a^{2}}{72 \pi(1-v)} \ln \frac{\sqrt{2} d}{a}, \widetilde{E}=\frac{G a^{2}}{72 \pi(1-v)}$.

between individual partials and twin boundaries. In addition, a higher stacking fault energy makes it easier for a dissociated perfect dislocation to constrict at the twin boundary before the leading partial reacts at the twin boundary. This is especially significant for perfect screw dislocations, which, once constricted, can cross-slip into the twin boundary plane or be transmitted across the twin boundary to glide on a slip plane in the twin, without any dislocation reaction.

If the stacking fault energy is very low partial dislocations can be emitted in large numbers from grain boundaries and other sources [52,53]. These partials can crossslip into the twin boundaries with small energy barriers (see Table 1), which generate steps on the twin boundaries. It can also be seen from Table 1 that the energy barriers for $90^{\circ}$ partials to be transmitted across twin boundaries are much lower than the energy barrier for $30^{\circ}$ partials to be transmitted across a twin boundary. In addition, a low stacking fault energy makes it difficult for a dissociated perfect dislocation to constrict. This could produce a scenario in which the leading partial cross-slipped into the twin boundary plane or was transmitted across the twin boundary, while the trailing partial remains on the original slip plane in the matrix. Such a dislocation structure is believed to be effective in improving the work hardening rate [9].

\section{Summary}

In this paper we have systematically described the plausible dislocation reactions at twin boundaries. Such reactions and subsequent dislocation slips determine how the twins affect the deformation behavior of nanocrystalline fcc metals. Plausible dislocation reactions and the energy barriers associated with the reactions are listed in Table 1. It should be noted that the equations used to calculate the dislocation elastic energies are based on isotropic elasticity. Therefore, the energy barriers listed in Table 1 are isotropic energy barriers. The energy barrier determines the feasibility of a dislocation reaction. A lower energy barrier indicates that the reaction is statistically more likely to occur. However, other factors, including the orientation and magnitude of the applied stress and the stacking fault energy also play important roles. It should be noted that most of the dislocation-twin reactions described in this paper may not occur in coarse grained fcc metals and alloys because of their requirement of high applied stresses to overcome the energy barriers.

\section{Acknowledgements}

Y.T.Z. were supported by the US Army Research Office (W911NF-09-1-0427) and Army Research Laboratory 
(W911QX-08-C-0083). X.L.W. acknowledges the support of NSFC Grant Nos. 10721202, 11072243, and 973 and Project Grants Nos. 2010CB631004 and 2009CB623700.

\section{References}

[1] Li XY, Wei YJ, Lu L, Lu K, Gao HJ. Nature 2010;464:877.

[2] Lu L, Shen YF, Chen XH, Qian LH, Lu K. Science 2004;304:422.

[3] Lu K, Lu L, Suresh S. Science 2009;324:349.

[4] Wang L, Han X, Liu P, Yue Y, Zhang Z, Ma E. Phys Rev Lett 2010;105:135501.

[5] Lu L, Dao M, Zhu T, Li J. Scripta Mater 2009;60:1062.

[6] Zhao YH, Zhu YT, Liao XZ, Horita Z, Langdon TG. Appl Phys Lett 2006;89:121906.

[7] Zhao YH, Liao XZ, Horita Z, Langdon TG, Zhu YT. Mater Sci Eng A 2008;493:123.

[8] Zhao YH, Bingert JE, Liao XZ, Cui BZ, Han K, Sergueeva AV, et al. Adv Mater 2006;18:2949.

[9] Wu XL, Zhu YT, Wei YG, Wei Q. Phys Rev Lett 2009;103:205504.

[10] Wang ZW, Wang YB, Liao XZ, Zhao YH, Lavernia EJ, Zhu YT, et al. Scripta Mater 2009;60:52.

[11] Liao XZ, Zhao YH, Srinivasan SG, Zhu YT, Valiev RZ, Gunderov DV. Appl Phys Lett 2004;84:592.

[12] Schiotz J, Di Tolla FD, Jacobsen KW. Nature 1998;391:561.

[13] Shan ZW, Stach EA, Wiezorek JMK, Knapp JA, Follstaedt DM, Mao SX. Science 2004;305:654.

[14] Van Swygenhoven H, Derlet PM, Hasnaoui A. Phys Rev B 2002;66:024101.

[15] Kumar KS, Suresh S, Chisholm MF, Horton JA, Wang P. Acta Mater 2003;51:387.

[16] Liao XZ, Kilmametov AR, Valiev RZ, Gao HS, Li XD, Mukherjee AK, et al. Appl Phys Lett 2006;88:021909.

[17] Asaro RJ, Suresh S. Acta Mater 2005;53:3369.

[18] Rice JR. J Mech Phys Solids 1992;40:239.

[19] Wang YM, Hodge AM, Biener J, Hamza AV, Barnes DE, Liu K, et al. Appl Phys Lett 2005;86:101915.

[20] Wu X, Zhu YT, Chen MW, Ma E. Scripta Mater 2006;54:1685.

[21] Wu XL, Liao XZ, Srinivasan SG, Zhou F, Lavernia EJ, Valiev RZ, et al. Phys Rev Lett 2008;100:095701.

[22] Wu XL, Zhu YT. Phys Rev Lett 2008;101:025503.

[23] Sennour M, Lartigue-Korinek S, Champion Y, Hytch MJ. Philos Mag 2007;87:1465.

[24] Sennour M, Lartigue-Korinek S, Champion Y, Hytch MJ. J Mater Sci 2008:43:3806
[25] Barry DE, Mahajan S. Philos Mag 1971;23:727.

[26] Mahajan S. Philos Mag 1971;23:781.

[27] Mahajan S. J Phys F 1972;2:19.

[28] Wang YB, Wu B, Sui ML. Appl Phys Lett 2008;93:041906.

[29] Wu XL, Narayan J, Zhu YT. Appl Phys Lett 2008;93:031910.

[30] Mullner P, Solenthaler C. Mater Sci Eng A 1997;230:107.

[31] Medlin DL, Carter CB, Angelo JE, Mills MJ. Philos Mag A 1997;75:733.

[32] Wang YB, Sui ML. Appl Phys Lett 2009;94:021909.

[33] Afanasyev KA, Sansoz F. Nano Lett 2007;7:2056.

[34] Jin ZH, Gumbsch P, Ma E, Albe K, Lu K, Hahn H, et al. Scripta Mater 2006;54:1163.

[35] Jin ZH, Gumbsch P, Albe K, Ma E, Lu K, Gleiter H, et al. Acta Mater 2008;56:1126.

[36] Yamakov V, Wolf D, Phillpot SR, Gleiter H. Acta Mater 2003;51:4135.

[37] Sansoz F, Huang HC, Warner DH. JOM 2008;60(9):79.

[38] Henager CH, Hoagland RG. Scripta Mater 2004;50:701.

[39] Jin J, Shevlin SA, Guo ZX. Acta Mater 2008;56:4358.

[40] Shabib I, Miller RE. Acta Mater 2009;57:4364.

[41] Froseth AG, Derlet PM, Van Swygenhoven H. Appl Phys Lett 2004;85:5863.

[42] Zhu YT, Narayan J, Hirth JP, Mahajan S, Wu XL, Liao XZ. Acta Mater 2009;57:3763.

[43] Kovács I, Zsoldos L. Dislocation and plastic deformation. Oxford (UK): Pergamon; 1973.

[44] Liao XZ, Srinivasan SG, Zhao YH, Baskes MI, Zhu YT, Zhou F, et al. Appl Phys Lett 2004;84:3564.

[45] Hirth JP, Lothe J. Theory of dislocations. Malabar (FL): Krieger Publishing Co.; 1992.

[46] Ichimura M, Narayan J. Philos Mag A Phys Condens Matter Struct Defects Mech Properties 1995;72:281.

[47] Zhu YT, Liao XZ, Valiev RZ. Appl Phys Lett 2005;86:103112.

[48] Liao XZ, Huang JY, Zhu YT, Zhou F, Lavernia EJ. Philos Mag 2003;83:3065

[49] Cao AJ, Wei YG. Appl Phys Lett 2006;89:041919.

[50] Zhu T, Li J, Samanta A, Kim HG, Suresh S. Proc Natl Acad Sci USA 2007; 104:3031.

[51] Dao M, Lu L, Shen YF, Suresh S. Acta Mater 2006;54:5421.

[52] Zhu YT, Liao XZ, Srinivasan SG, Zhao YH, Baskes MI, Zhou F, et al. Appl Phys Lett 2004;85:5049.

[53] Zhu YT, Liao XZ, Srinivasan SG, Lavernia EJ. J Appl Phys 2005;98:034319. 